\title{
Derrame pericárdico como manifestación clínica única de lupus eritematoso sistémico
}

\author{
M. BLANCO RAMOS, M. A. CAÑIZARES CARRETERO, E. M. GARCÍA-FONTÁN, \\ J. E. RIVO VÁZQUEZ
}

Servicio de Cirugía Torácica. Complexo Hospitalario Xeral-Cíes. Vigo. Pontevedra

\section{RESUMEN}

La afectación del pericardio es la manifestación cardíaca más frecuente del lupus eritematoso sistémico (LES). Sin embargo, es infrecuente que el derrame pericárdico sea la forma de presentación del LES. Describimos el caso de un paciente varón con LES que debutó clínicamente con derrame pericárdico, comentamos su curso clínico y revisamos en la bibliografía médica distintas formas de presentación del LES.

PALABRAS CLAVE: Derrame pericárdico. Lupus eritematoso sistémico.

Blanco Ramos M, Cañizares Carretero MA, García-Fontán EM, Rivo Vázquez JE. Derrame pericárdico como manifestación clínica única de lupus eritematoso sistémico. An Med Interna (Madrid) 2006; 23: 80-82.

\begin{abstract}
Pericarditis is the most frequent cardiac manifestation of systemic lupus erythematosus (SLE), but pericardial effusion rarely occurs as the only first clinical manifestation of SLE. We report the case of a male patient whose initial manifestation of lupus was a pericardial effusion. We comment on its clinical course and we reviewed in the medical literature different presenting features in SLE.
\end{abstract}

KEY WORDS: Pericardial effusion. Systemic lupus eythematosus.
PERICARDIAL EFFUSION AS THE ONLY FIRST CLINICAL MANIFESTATION OF SYSTEMIC LUPUS ERYTHEMATOSUS

\section{INTRODUCCIÓN}

El lupus eritematoso sistémico (LES) es una enfermedad inflamatoria multisistémica que se caracteriza por la presencia de fenómenos de autoinmunidad incluyendo reactividad para anticuerpos antinucleares, el ADN de doble cadena y el antígeno Sm (1). La afectación cardíaca constituye una de las manifestaciones clínicas más comunes de la enfermedad (2), siendo una de las principales causas de muerte en pacientes de larga evolución (3).

La pericarditis fue la primera manifestación cardíaca reconocida del LES y ha sido referida también como una de las complicaciones más frecuentes (uno de cada cuatro pacientes pueden llegar a presentarla). La prevalencia de la pericarditis como manifestación del LES es del 12 al $48 \%$ en adultos, y en niños de un 5 a un $25 \%$ (4). Se manifiesta clínicamente por dolor precordial o subesternal y de tipo posicional algunas veces, con una intensidad moderada a severa. Sin embargo, en un $62 \%$ de casos se ha descrito la existencia de derrame pericárdico sin la presencia de pericarditis (5).
Se describe un caso clínico de LES con confirmación diagnóstica clínica e inmunológica que debutó clínicamente como única manifestación con derrame pericárdico.

\section{CASO APORTADO}

Un varón de 77 años no fumador y con antecedentes de síndrome restrictivo por neumoconiosis y tromboembolismo pulmonar multisegmentario bilateral, acudió al servicio de urgencias de nuestro hospital presentando un cuadro de malestar y debilidad muscular. Del interrogatorio se recogió un deterioro progresivo del estado general un mes después de haber sido dado de alta por una neumonía del lóbulo inferior derecho con derrame pleural paraneumónico. Se acompañaba de sudoración profusa y fatiga.

En el examen físico se verificó una presión arterial de 95/60 $\mathrm{mmHg}$, temperatura axilar de $39,5{ }^{\circ} \mathrm{C}$, frecuencia cardíaca 115 lat/min, frecuencia respiratoria 35, auscultación cardíaca rítmica, crepitantes basales en la auscultación pulmonar, hepatomegalia de cuatro traveses, sin edemas en miembros. La exploración complementaria efectuada al ingreso reveló una velocidad de sedimentación

Trabajo aceptado: 21 de septiembre de 2005

Correspondencia: Montserrat Blanco Ramos. Servicio de Cirugía Torácica. Complexo Hospitalario Xeral-Cíes. C/ Pizarro, 22. 36204 Vigo. Pontevedra. e-mail: montsebr@usc.es 
de 105, hemoglobina 12,8 g/dl, VCM 81, leucocitos 6350/ $\mu 1,244$ mil plaquetas/ $\mu 1$, actividad de protrombina $53,8 \%$, urea 56 , ácido úrico 1,7, colesterol 109, albúmina 2,88 g, Ca 8, LDH 523, T4 1,08, TSH 4,71, siendo el resto normal. En el ECG se observó ritmo sinusal a 98 lat/min, bajo voltaje y trastornos inespecíficos de repolarización. La radiografía de tórax mostró un pequeño derrame pleural bilateral. En la TC torácica (Fig. 1) se pudo verificar el derrame pleural bilateral, atípico con loculaciones, y un derrame pericárdico de moderada cuantía. Se le practicó por ello un estudio ecocardiográfico que fue informado como ausencia de signos de taponamiento, una dilatación moderada de la aurícula izquierda, un ventrículo izquierdo con hipertrofia concéntrica, una FEV1 del 60\%, alteración de la relajación del ventrículo izquierdo y despegamiento ligero del pericardio de todos los segmentos salvo a nivel infero-posterior. Las válvulas eran normales, con pequeñas placas de calcio en la válvula mitral. Se decidió realizar una toracocentesis, obteniéndose un exudado con $67 \%$ de PMN, 33\% de mononucleares, glucosa 90, proteínas totales 4,3, pH 7,34, ADA 25, LDH 363. Sin gérmenes en el gram, BAAR negativo, cultivo Lowestein negativo y citología negativa. Una radiografía de manos mostró cambios en relación con osteoartritis primaria muy evolucionada, afectándose ambas manos, sobre todo las articulaciones interfalángicas distales y metacarpofalángicas. Dados los datos clínicos y la existencia de derrame pericárdico, a pesar de la ausencia de signos indicativos de taponamiento cardíaco, se decidió practicar una ventana pericárdica mediante minitoracotomía anterior izquierda con intención diagnóstica, obteniéndose muestras de pericardio parietal, así como unos 150 cc de líquido pericárdico. El estudio anatomopatológico demostró una inflamación leve, sin evidencia de células neoplásicas.

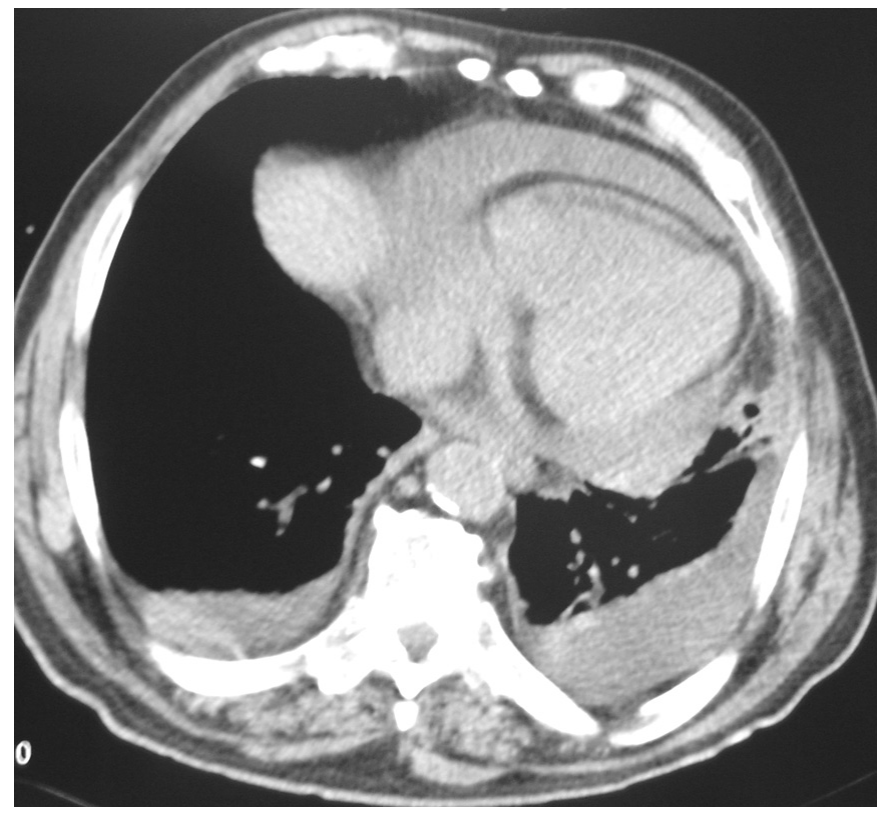

Fig. 1.

Dado que los resultados obtenidos en las exploraciones complementarias fueron poco concluyentes, se decidió realizar una batería de determinaciones inmunológicas que incluía ANA, antiENA, anti-SSA, anti-ADN, anti-ANCA, anti-SSB, anti-RNP, y anti-scl. De ellos, fueron considerados como positivos los ANA (1/320 con patrón homogéneo), los anti-ENA y los anti-SSA. Las cifras de C3 y C4 fueron respectivamente $146 \mathrm{mg} / \mathrm{dl}$ y $28 \mathrm{mg} / \mathrm{dl}$. Los hallazgos obtenidos condujeron al diagnóstico de LES. Se inició tratamiento con prednisona $10 \mathrm{mg} / 12 \mathrm{~h}$, produciéndose mejoría clínica y remisión de la fiebre. En los controles posteriores ecocardiográficos se observó una total desaparición del derrame pericárdico.

\section{DISCUSIÓN}

El LES se manifiesta predominantemente en mujeres después de la pubertad, siendo la manifestación clínica más frecuente la afectación articular. Otra sintomatología acompañante suele ser el rash malar en alas de mariposa, la nefritis lúpica, serositis o mononeuritis sensitiva (6). Se han descrito en la literatura otras formas de debut del LES, como pancreatitis (7), hemorragia pulmonar (8), trombosis venosa central (9) o derrame pleural bilateral masivo (10). La presentación como derrame pericárdico se produce en un 1-2\% de los casos, y sólo un $0,8 \%$ se inicia como taponamiento cardíaco (11).

Los criterios clínicos del LES son descritos en la Tabla I. El diagnóstico viene dado por la coexistencia de cuatro de estos criterios $(12,13)$. De ellos, en nuestro caso, solamente se encontraba presente el derrame pericárdico como característica clínica, teniendo en cuenta que la etiología es muy variable (12) (Tabla II). A pesar del bajo índice de sospecha, se procedió a las determinaciones analíticas que llevaron al diagnóstico (ANA y anti-SSA).

\section{TABLA I}

\section{CRITERIOS CLINICOS DIAGNÓSTICOS DE LUPUS ERITEMATOSO SISTÉMICO}

\begin{tabular}{l} 
1. Exantema \\
Malar \\
Discoide \\
2. Serositis (pleuritis o pericarditis) \\
Pleuritis \\
Pericarditis \\
3. Úlceras orales \\
4. Clínica neurológica sin otra causa conocida alteracio- \\
nes inmunológicas (células LE, Ac anti-ADN, anti-SM, \\
VDRL) \\
Convulsiones \\
Psicosis \\
5. Artritis no erosiva \\
6otosensibilidad \\
7. Afectación renal \\
Proteinuria \\
Cilindros celulares \\
8. Alteraciones hematológicas \\
Anemia \\
Leucopenia \\
Trombopenia \\
9. Alteraciones inmunológicas \\
Células LE \\
Anticuerpos anti-ADN \\
Anticuerpos anti-Sm \\
VDRL (falso positivo) \\
10. ANA positivos \\
\hline
\end{tabular}

Aunque la afectación cardiaca en el LES es infrecuente, la introducción de la ecocardiografía en el arsenal diagnóstico habitual ha hecho aumentar la identificación de derrames pericárdicos asintomáticos hasta en un $48 \%$ de los casos (14). No obstante, está descrito en la literatura que el taponamiento cardíaco puede ser la primera manifestación de LES (14-16).

El tratamiento de elección del derrame pericárdico por LES consiste en prednisona a dosis altas. En caso de tapona- 
TABLA II

FACTORES ETIOLÓGICOS DEL DERRAME PERICÁRDICO (POR ORDEN DE FRECUENCIA)

1. Pericarditis idiopática
2. Pericarditis vírica
3. Pericarditis tuberculosa
4. Pericarditis purulenta
5. Pericarditis de enfermedades auntoinmunes y vasculitis
6. Pericarditis secundaria a afectación de estructuras pró-
$\quad$ ximas
7. Pericarditis asociadas a enfermedades metabólicas
8. Pericarditis neoplásica

1. Pericarditis idiopática

2. Pericarditis vírica

3. Pericarditis tuberculosa

4. Pericarditis purulenta

5. Pericarditis de enfermedades auntoinmunes y vasculitis

. Pericarditis secundaria a afectación de estructuras pró-

7. Pericarditis asociadas a enfermedades metabólicas

8. Pericarditis neoplásica

\section{Bibliografía}

1. Reeves WH, Satoh M. Features of autoantigens. Mol Biol Rep 1996; 23: 217-226.

2. Ansari A, Larson PH, Bates HD. Cardiovascular manifestations os systemic lupus erythematosus: current perspective. Prog Cardiovasc Dis 1985; 27: 421-434

3. Petri M, Buyon J, Kim M. Classification and definition of major flares in SLE clinical trials. Lupus 1999; 8: 685-691.

4. Moder KG, Miller TD, Tazelaar HD. Cardiac involvement in systemic lupus erythematosus. Mayo Clin Proc 1999; 74: 275-284.

5. Doherty NE, Siegel RJ. Cardiovascular manifestations of systemic lupus erythematosus. Am Heart J 1985; 110: 1257-1265.

6. Wallace DJ. The clinical presentation of systemic lupus erythematosus. En: Wallace DJ, Hahn BH, editors. Dubois' lupus erythematosus. 5th ed. Baltimore: Lippincott Wilkins \& Wilkins. 1997. p. 627.

7. Duncan HV, Achara G. A rare initial manifestation of systemic lupus erythematosus-acute pancreatitis: case report and review of the literature. J Am Board Fam Pract 2003; 16: 334-338.

8. Hernández-Beriain JA, González T, Rodríguez-Lozano B, Mora ML. Massive pulmonary hemorrhage as early manifestation of systemic lupus erythematosus. Therapeutic possibilities. Med Clin (Barc) 1992; 99: 716-717.

9. Cardona-Portela P, Casasnovas-Pons C, Moral-Torres M, Rubio-Borre- miento cardíaco es necesario realizar una pericardiocentesis para aliviar el grave compromiso hemodinámico y obtener una muestra de líquido pericárdico con el fin de intentar llegar a un diagnóstico. El 20\% de los pacientes no responden adecuadamente al tratamiento corticoide y tienen una recurrencia del taponamiento pericárdico que requiere una pericardiotomía en algún momento de la enfermedad (11).

Como conclusión, este caso clínico ilustra el interés de considerar el derrame pericárdico sin taponamiento ni signos de pericarditis como posible manifestación de un LES, con el fin de realizar la batería diagnóstica pertinente. Esto permitiría iniciar el tratamiento corticoide de la enfermedad precozmente y prevenir situaciones de compromiso hemodinámico.

go F. Cerebral venous thrombosis as the presenting symtom of systemic lupus erythematous. Rev Esp Neurol 2004; 39: 30-34.

10. Bouros D, Panagou P, Papandreou L, Kottakis I, Tegos C. Massive bilateral pleural effusion as the only first presentation of systemic lupus erythematosus. Respiration 1992; 59: 173-175.

11. Estes D, Christian CL. The natural history of systemic lupus erythematosus by prospective analiysis. Medicine 1971; 50: 85-90.

12. Farreras-Rozman. Enfermedades del pericardio. En: Medicina Interna $13^{\mathrm{a}}$ edición. Mosby-Doyma 1995; 1: 582-583.

13. Font J, Cervera R. Ten years of new criteria for the classification of systemic lupus erythematosus. Is everything clear? Med Clin (Barc) 1992; 99: 738-740.

14. Manresa JM, Gutiérrez L, Viedma P, Alfani O. Taponamiento cardíaco como presentación clínica de lupus eritematoso sistémico. Rev Esp Cardiol 1997; 50: 600-602.

15. Kelly TA. Cardiac tamponade in systemic lupus erythematosus: an unusual initial manifestation. South Med J; 1987: 514-516.

16. Arostegui Lavilla J, Garcia Vivar ML, Galíndez Agirregoikoa E, García Llorente JF, Aranburu Albizuri JM. Cardiac tamponade as initial manifestation of systemic lupus erythematosus. An Med Interna (Madrid) 2001; 18: 451-452. 